\title{
The banding of an esophageal varix
}

\author{
Robert Enns MD FRCP
}

T he proportion of patients with upper gastrointestinal bleeding (UGI) from esophageal varices varies among centres; however, in approximately $10 \%$ of all emergency patients with UGI bleeding, the source is identified as esophageal varices (1). Centres that have a special interest in liver disease may have a higher proportion of patients presenting with this problem. The significance of these lesions is highlighted by the high morbidity and mortality rates associated with the presence of acutely bleeding varices. Although varices from portal hypertension may develop within the stomach (ie, fundal), small intestine (duodenal, jejunal or ileal) or colon (classically rectal), the most common site is within the esophagus. The etiology of the varices is usually cirrhosis; however, any cause of portal hypertension (ie, portal vein thrombosis) can theoretically result in a similar vascular outcome.

Overall, approximately $60 \%$ of patients with cirrhosis develop esophageal varices and $60 \%$ of these have a bleeding episode (2). The natural history of esophageal varices is dramatic in that up to one-third of patients may rebleed over the subsequent six weeks if untreated. The significance of this problem has therefore spawned numerous studies, and the optimal management of bleeding esophageal varices has been determined.

The first step in the management of any UGI bleeding patient is resuscitation. As endoscopic therapy has become more widely available, the urgent call for the endoscopist tends be rapidly transmitted with alarming speed from the emergency room when an unstable patient arrives with hematemesis and/or melena. It should be clearly recognized that in the 'ABCs' of resuscitation, the 'E' for endoscopy actually comes after airway, breathing and circulation have been adequately managed. Most patients with UGI bleeding (approximately $80 \%$ ) stabilize with intravenous fluid resuscitation. Access with two large-bore intravenous lines is preferable so that pharmacological agents and blood transfusions may be simultaneously administered.

In the setting of esophageal variceal hemorrhage, the use of ancillary medications to improve outcomes (primarily by decreasing portal pressures) has been advocated. For many years, vasopressin (with or without nitroglycerin) was used at a dose of 0.4 units/min. Morbidity from the vasopressin occurs in $20 \%$ of patients if it is used in isolation; therefore, the addition of nitroglycerin has been instituted to decrease this risk.
Another approach to avoid complications from vasopressin is to alter its underlying structure, so that a 'slow-release' vasopressin analogue results (glypressin). Glypressin has been associated with fewer side effects, but without improved efficacy.

Unlike vasopressin, octreotide has no effect on systemic arterial pressure, cardiac index or pulse rate. Somatostatin and its longer-acting analogue octreotide are presently the primary pharmacological modality of therapy for UGI bleeding from esophageal varices. Somatostatin has a half-life of $2 \mathrm{~min}$ to $3 \mathrm{~min}$, with a pharmacological half-life of $90 \mathrm{~min}$. A bolus of $75 \mu \mathrm{g}$ to $100 \mu \mathrm{g}$ decreases splanchic blood flow by $25 \%$, total hepatic blood flow by $25 \%$, wedged hepatic vein pressure by $10 \%$ and measured varix pressure by approximately $35 \%$ (3-5).

Somatostatin has been compared with vasopressin/glypressin with no significant difference in mortality; however, overall, there appears to be a decrease in the rebleeding rate with a lower complication rate of therapy $(3,6-10)$. In at least one Cochrane meta-analysis (5), it has been suggested that vasoactive drugs (particularly octreotide/somatostatin) were more effective in isolation than sclerotherapy, with fewer side effects, and therefore, sclerotherapy should be reserved only for those failing medical management.

Typically, for those patients with a high suspicion of variceal hemorrhage, the use of octreotide $(50 \mu \mathrm{g}$ bolus with $50 \mu \mathrm{g} / \mathrm{h})$ is initiated before the endoscopic examination. Recently, the addition of an intravenous proton pump inhibitor has also been advocated for patients with significant UGI bleeding; however, although there is theoretical benefit of acid suppression in any source of UGI bleeding (by increasing $\mathrm{pH}$, one improves platelet aggregation and decreases fibrinolysis), the clinical benefit in the setting of esophageal variceal hemorrhage remains to be proven.

The key to any therapeutic endoscopic procedure is adequate preparation. This includes appropriately trained nursing staff (ideally, two nurses assisting in the endoscopy suite), a selection of different endoscopes (particularly therapeutic endoscopic channels to allow adequate suction and irrigation) and an array of therapeutic accessories (injection catheters, clips, bicap cautery). The instruments should be easily available, and a full understanding of their function and assembly is critical. Too often, the physician relies upon the nurse to 'make this work' without understanding the true limitations of various devices. Clearly, well-trained endoscopy nurses are critical, 


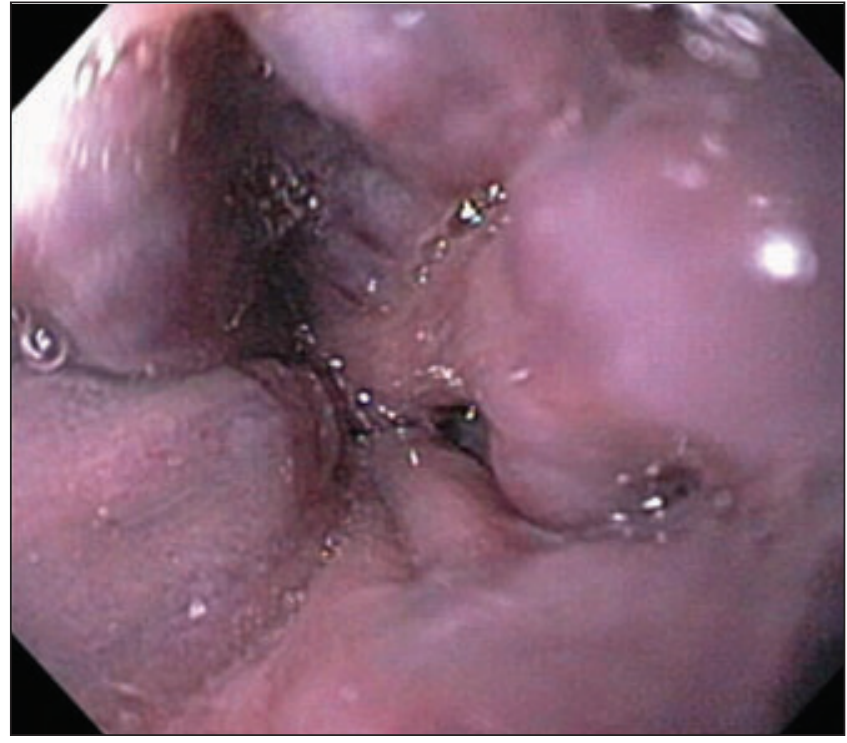

Figure 1) Bulging esophageal varices in the distal esophagus

particularly with therapeutic cases; however, the responsibility of the patient still rests on the physician, and therefore, adequate understanding of the equipment enables a more confident, expedient approach.

Although elective endoscopic variceal band ligation can usually be performed quickly, the scheduling of acute, severe UGI bleeding should be appropriate to ensure that neither nurse nor physician is unduly pressured. Sedation is provided in standard fashion; in general, a well-sedated patient is ideal for technically challenging procedures. The endoscope is inserted and slowly advanced into the esophagus. If there are varices present, the presence of active spurting, red or white 'wale' signs or large varices are all negative prognostic markers. If the site of active bleeding is definitively isolated within the esophagus (ie, spurting), then I will note the endoscopic distance, and may remove the endoscope and immediately apply a variceal ligator apparatus. Because usually only one site is bleeding, if that site can be localized at reinsertion and banded, the episode of bleeding is theoretically treated. If, upon re-entry, the site of spurting is obliterated, at least I have already determined the depth of penetration of the instrument where the hemorrhage site was present and I can concentrate my 'cleaning' efforts at that distance.

If no site of active bleeding is noted, an examination of the stomach and duodenum is performed to ensure there are no other areas of abnormality, and the esophagus is once more evaluated on withdrawal of the instrument. The distance (from the incisors) to the gastroesophageal junction is carefully measured. Because most bleeding from varices occurs within the distal $3 \mathrm{~cm}$ to $4 \mathrm{~cm}$ of the esophagus, this is the critical area to concentrate therapy upon (Figure 1). When evaluating this area, I carefully plan my first two or three banding sites at locations where the varices appear the most prominent. I plan this before I attach the banding apparatus because visibility is slightly decreased when the banding device is applied to the endoscope (Figure 2).

There have been a number of studies comparing endoscopic band ligation with endoscopic sclerotherapy, most demonstrating decreased complication rates of band ligation without a sacrifice of outcomes. The role for endoscopic sclerotherapy,

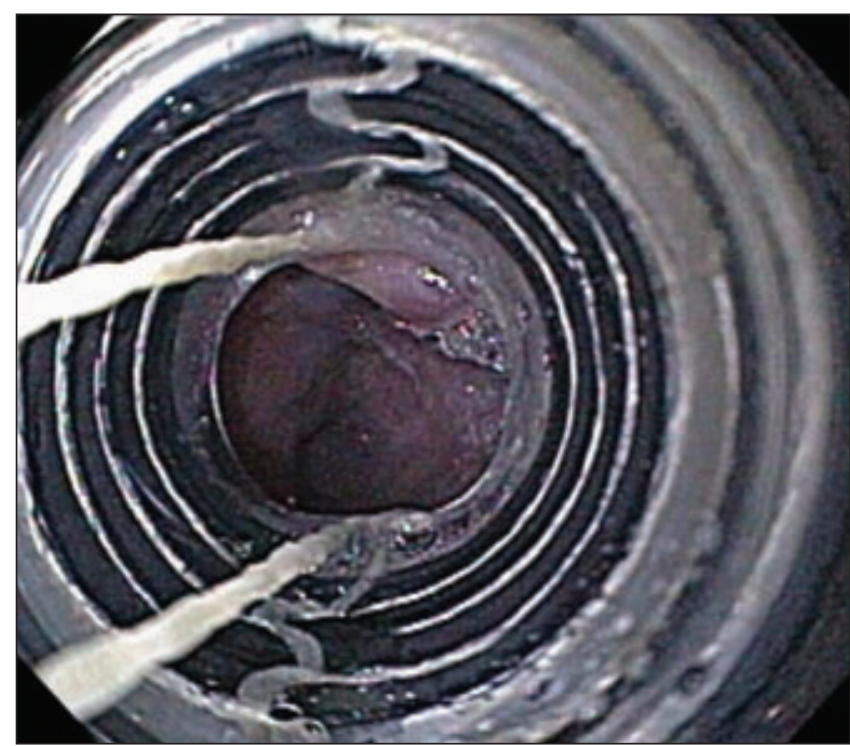

Figure 2) Banding visualization is slightly decreased, and therefore, to simplify the banding, it is important to identify landmarks before insertion of the device

therefore, appears to be somewhat limited to rare events, in which obliteration of the endoscopic field by blood may force a 'blind' (yet controlled) four-quadrant injection of sclerosant in an effort to 'gain control'. The use of histocryl is usually not required for esophageal varices and is instead reserved for fundal varices $(11,12)$. Although detachable endoscopic snares have been used in esophageal and fundal varices, they are also not usually required $(13,14)$. There are a number of different multibanders available; all function with the same principle. They come in different numbers and styles and have been demonstrated to be safer and quicker, and require less sedation for the patient (15-20). At least six bands should be available for the initial endoscopic therapeutic session. Because it can be difficult to place more than approximately one band per centimetre of the distal esophagus, and we know that most bleeding is in the distal $4 \mathrm{~cm}$ to $6 \mathrm{~cm}$, usually six bands suffice for each treatment session.

The development of the multibander has supplanted the need for an overtube, which was responsible for many complications. The insertion of the banding device, however, is still not without hazard. The device attaches firmly onto the end of the instrument but is slightly larger and less tapered than the tip of the endoscope; insertion is therefore slightly more cumbersome. Upon entering the esophagus, I usually adjust the device wheel to the 'firing' position so that I am ready to band. The endoscope is advanced to the predetermined distance, and I attempt to locate the variceal site. The key to a successful banding procedure is often the careful placement of the first band. Once localized, suction is applied, and when adequate tissue engulfs the chamber (visible endoscopically), the first band is applied (Figures 3 and 4). If an adequate amount of tissue is not engaged, often a gentle 'jiggle' of the endoscope enables adjacent vascular/ esophageal tissue to be brought into the bander for entrapment. Following the application of the first band, the second site is identified and the process repeated. If the procedure is without complication, all bands are applied and it is usually not necessary to pass distally to them endoscopically again, because a risk of 'traumatizing' them theoretically exists. 


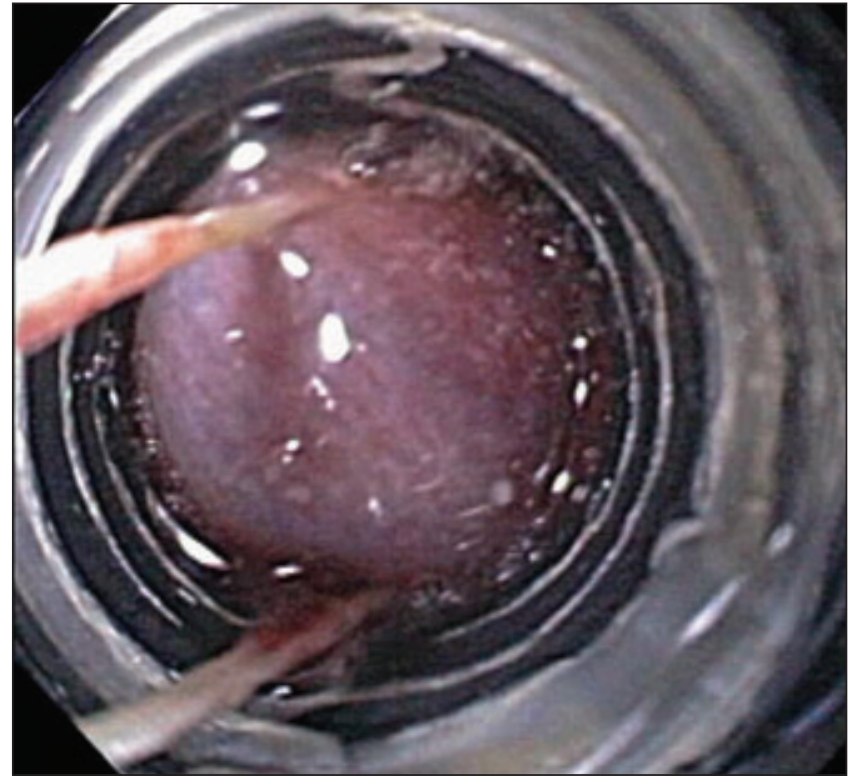

Figure 3) Suction is applied to the varix and a bulge into the chamber is endoscopically visible, at which time the band is applied. A slight 'jiggle' of the endoscope can encourage tissue to advance into the chamber for banding

If the bands have been applied distally, the proximal vascular markings usually diminish dramatically (because the blood flow is cephalad in esophageal varices), limiting the necessity to use large numbers of bands. Repeat endoscopic assessment and banding is usually performed two to four weeks later. Often, three to four endoscopic sessions are required to obliterate esophageal varices.

To guide aggressiveness of therapy, a number of predictors of variceal bleeding have been determined. These include the

\section{REFERENCES}

1. Silverstein FE, Gilbert DA, Tedesco FJ, Buenger NK, Persing J. The national ASGE survey on upper gastrointestinal bleeding. I. Study design and baseline data. Gastrointest Endosc 1981;27:73-9.

2. Baker LA, Smith C, Lieberman G. The natural history of esophageal varices; a study of 115 cirrhotic patients in whom varices were diagnosed prior to bleeding. Am J Med 1959;26:228-37.

3. Cello JP. Endoscopic management of esophageal variceal hemorrhage: Injection, banding, glue, octreotide, or a combination? Semin Gastrointest Dis 1997;8:179-87.

4. Corley DA, Cello JP, Adkisson W, Ko WF, Kerlikowske K. Octreotide for acute esophageal variceal bleeding: A meta-analysis.

Gastroenterology. 2001;120:946-54.

5. D’Amico G, Pietrosi G, Tarantino I, Pagliaro L. Emergency sclerotherapy versus vasoactive drugs for variceal bleeding in cirrhosis: A Cochrane meta-analysis. Gastroenterology 2003;124:1277-91.

6. de Franchis R. Somatostatin, somatostatin analogues and other vasoactive drugs in the treatment of bleeding oesophageal varices. Dig Liver Dis 2004;36(Suppl 1):S93-100.

7. Hwang SJ, Lin HC, Chang CF, et al. A randomized controlled trial comparing octreotide and vasopressin in the control of acute esophageal variceal bleeding. J Hepatol 1992;16:320-5.

8. Law AW, Gales MA. Octreotide or vasopressin for bleeding esophageal varices. Ann Pharmacother 1997;31:237-8.

9. Mezick SL, Henderson RP. Is somatostatin effective in the treatment of acute bleeding caused by esophageal varices? Ann Pharmacother 1994;28:739-40.

10. Zhou Y, Qiao L, Wu J, Hu H, Xu C. Comparison of the efficacy of octreotide, vasopressin, and omeprazole in the control of acute bleeding in patients with portal hypertensive gastropathy: A controlled study. J Gastroenterol Hepatol 2002;17:973-9.

11. Kiyosue H, Matsumoto S, Yamada Y, et al.Transportal intravariceal sclerotherapy with N-butyl-2-cyanoacrylate for gastric varices. J Vasc Interv Radiol 2004;15:505-9.

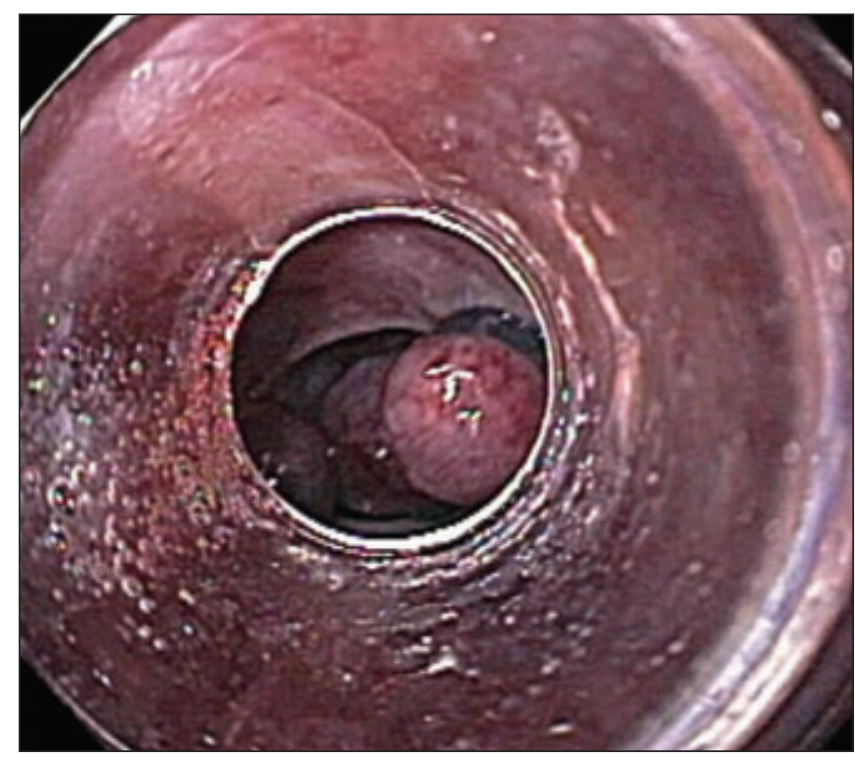

Figure 4) When all the bands have been released, the attaching 'string' is no longer visible. A cluster of banded varices are visible distally with bleeding now controlled

severity of liver disease (as measured by the Child classification system), size of varices, red colour signs (red wale sign), cherryred spots and hematocytic spots $(21,22)$. I have not discussed the issues of coagulation abnormalities (the use of vitamin $\mathrm{K}$ or frozen plasma), treatment of encephalopathy, antibiotic prophylaxis for patients with tense abdominal ascites and treatment of the underlying condition (ie, liver disease), all of which are paramount in the medical management of these patients.

12. Dhiman RK, Chawla Y, Taneja S, Biswas R, Sharma TR, Dilawari JB. Endoscopic sclerotherapy of gastric variceal bleeding with N-butyl-2cyanoacrylate. J Clin Gastroenterol 2002;35:222-7.

13. Shim CS, Cho JY, Park YJ, et al. Mini-detachable snare ligation for the treatment of esophageal varices. Gastrointest Endosc 1999;50:673-6.

14. Sung JJ, Chung SC. The use of a detachable mini-loop for the treatment of esophageal varices. Gastrointest Endosc 1998;47:178-81.

15. McKiernan PJ, Beath SV, Davison SM. A prospective study of endoscopic esophageal variceal ligation using a multiband ligator. J Pediatr Gastroenterol Nutr 2002;34:207-11.

16. Maekawa S, Aoyama N, Shirasaka D, Kasuga M. Endoscopic variceal scleroligation therapy with a newly designed multiple-band ligator device with special groove. Endoscopy 2004;36:378.

17. Wong T, Pereira SP, McNair A, Harrison PM. A prospective, randomized comparison of the ease and safety of variceal ligation using a multiband vs. a conventional ligation device. Endoscopy 2000;32:931-4.

18. Ell C, May A, Wurster $\mathrm{H}$. The first reusable multiple-band ligator for endoscopic hemostasis of variceal bleeding, nonvariceal bleeding and mucosal resection. Endoscopy 1999;31:738-40.

19. Saeed ZA. The Saeed Six-Shooter: A prospective study of a new endoscopic multiple rubber-band ligator for the treatment of varices. Endoscopy 1996;28:559-64.

20. Sackmann M, Gerbes AL. Application of a multiple-band ligator in active variceal bleeding. Endoscopy 1996;28:533.

21. Beppu K, Inokuchi K, Koyanagi N, et al. Prediction of variceal hemorrhage by esophageal endoscopy. Gastrointest Endosc 1981;27:213-8.

22. The Northern Italian Endoscopic Club for the Study and Treatment of Esophageal Varices. Prediction of the first variceal hemorrhage in patients with cirrhosis of the liver and esophageal varices: A prospective multicenter study. N Engl J Med 1988;319:983-9. 


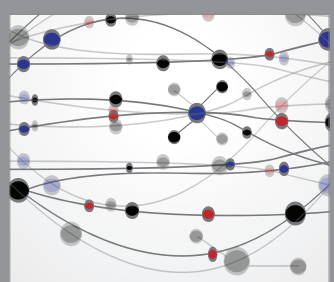

The Scientific World Journal
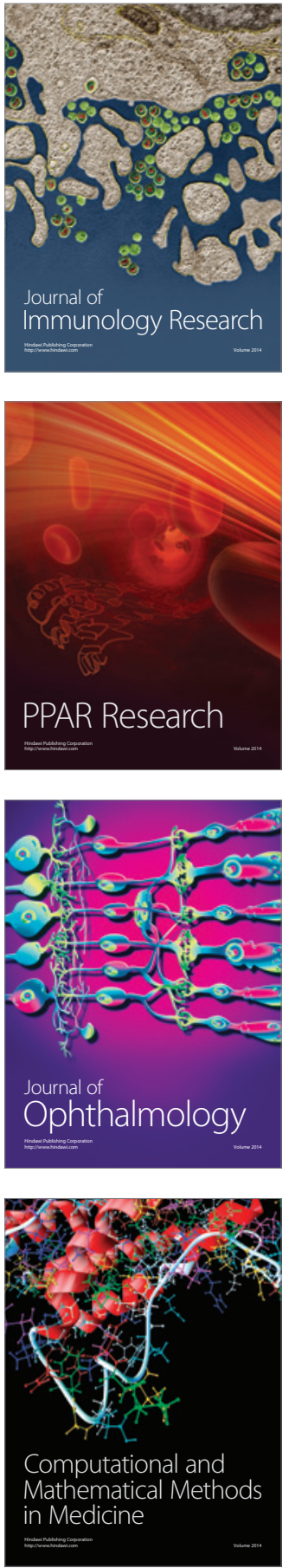

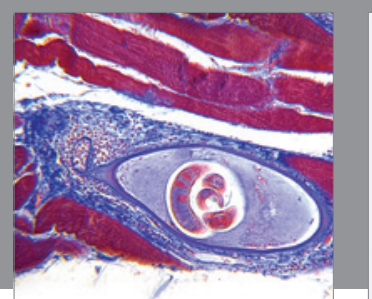

Gastroenterology Research and Practice

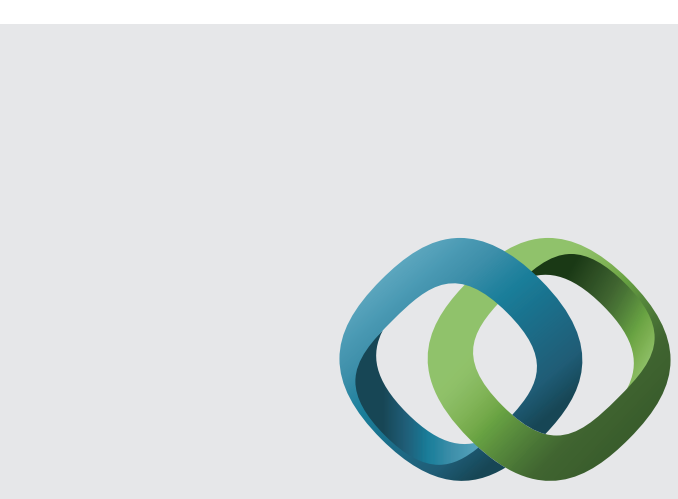

\section{Hindawi}

Submit your manuscripts at

http://www.hindawi.com
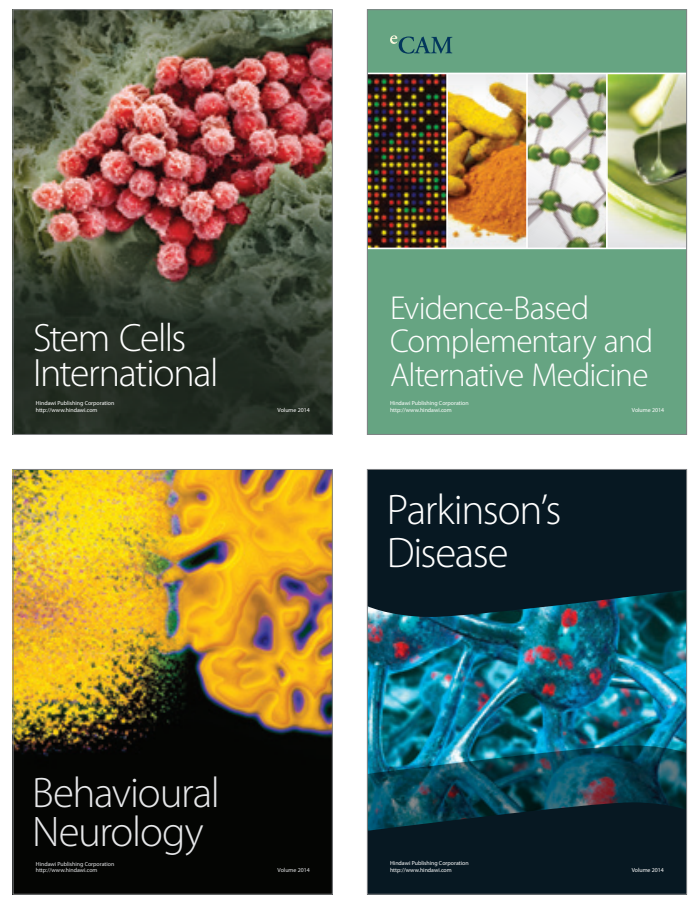
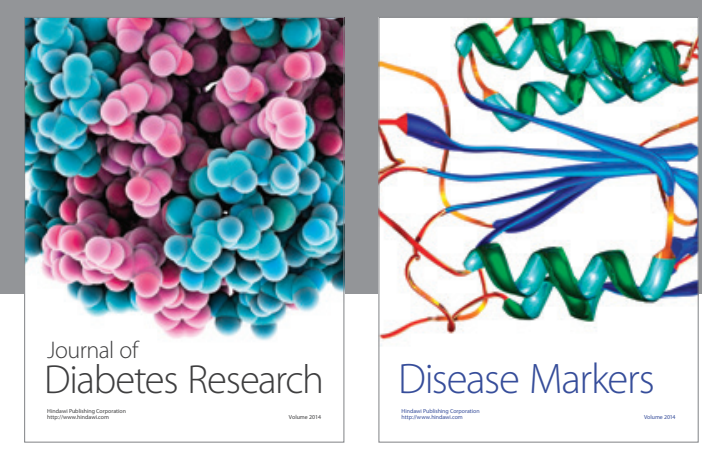

Disease Markers
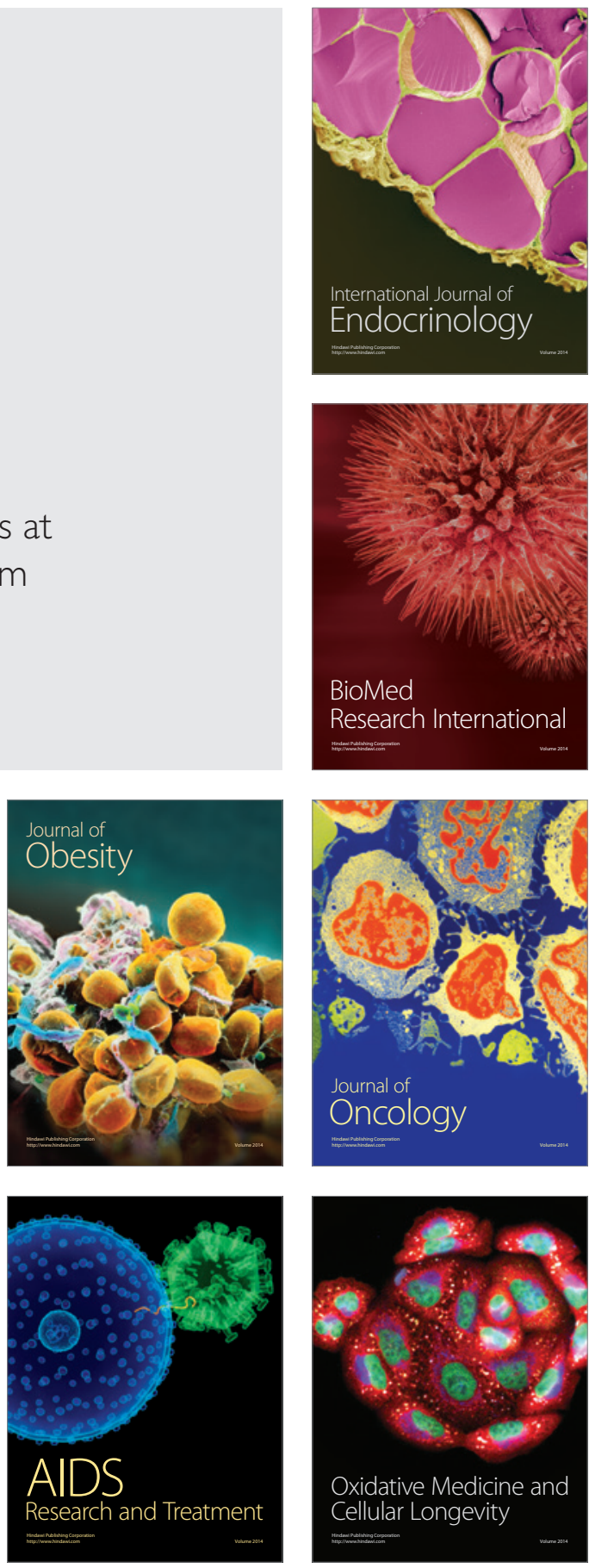